\title{
Effect of nano CdS dispersion on thermal conductivity of PS/PVC and PS/PMMA polymeric blend nanocomposites
}

\author{
Vishal Mathur · Dinesh Patidar · Kananbala Sharma
}

Received: 30 July 2014/ Accepted: 10 September 2014/Published online: 21 September 2014

(C) The Author(s) 2014. This article is published with open access at Springerlink.com

\begin{abstract}
The effect of dispersion of CdS nano-filler particles in respective PS/PVC and PS/PMMA polymer blend matrices on the effective thermal conductivity has been studied through Hot Disk Thermal Constant Analyzer based on transient plane source (TPS) technique. The thick film samples have been prepared by dispersing nano-filler particles of CdS (6 wt\%) in respective PS/PVC and PS/ PMMA binary blend matrices. The nanocomposite nature of prepared samples ascertained through small angle X-ray scattering (SAXS) as well as transmission electron microscopy (TEM) measurements. It is observed that at room temperature nano $\mathrm{CdS}$ dispersed polymeric blend samples offer higher effective thermal conductivity.
\end{abstract}

Keywords Polymer nanocomposite Effective thermal conductivity $\cdot$ Transient plane source method

\section{Introduction}

We know the polymers, polymer blends, polymer composites and filled polymers form the basis of polymer material science-the science of materials, investigation methods and control of their properties. In this regard, the nanocomposite of $\mathrm{CdS}$ can provide the promising possibilities to provide combinations of functionalities, such as thermally conducting composites with good mechanical

V. Mathur $(\bowtie)$

Faculty of Science and Technology, The ICFAI University,

Jaipur, India

e-mail: wishalmathur@gmail.com

V. Mathur · D. Patidar · K. Sharma

Semi-conductor and Polymer Science Laboratory, Department of

Physics, University of Rajasthan, Jaipur, India properties that are optically clear. Such properties can result because CdS nanoparticles, with diameters distinctly below the Rayleigh scattering limit, still display their solidstate physical properties when embedded in transparent matrices (Jung-Chul et al. 2009; Chen et al. 2007; Rong et al. 2003).

It is observed that literature of polymer research experiences insufficient efforts in the field of study of thermal transport properties. Agari et al. (1997) reported the thermal diffusivity and conductivity of Polystyrene/Polyphenylene oxide (PS/PPO) blends. DSC studies of blends confirm that the PS/PPO blends are miscible. The thermal diffusivity, specific heat capacity, density and thermal conductivity of the blends were measured in temperature range from the glass to liquid states. Agari et al. (1997) reported the study of thermal diffusivity and conductivity of Poly methyl methacrylate/Polycarbonate (PMMA/PC) blends. They found that thermal diffusivity and conductivity of the blend decreased slightly with increasing temperature to $440 \mathrm{~K}$ and then decreased abruptly in the lower critical solution temperature (LCST) range.

Actually the knowledge of thermal properties is required prior to any potential application as the environmental and thermal stability of the material should be known to identify the optimal condition for the performance of a device. The thermal conductivity is that thermal property which is required to describe the heat transfer through the material (Patidar and Saxena 2013).

In the continuation of our previously published research work (Mathur et al. 2009, 2013; Mathur and Sharma 2013), which was based on mechanical study of some thermoplastic polymeric blends like PS/PVC, PS/ PMMA, etc., and in view of the above facts, we realize the importance of studies of thermal properties of such polymeric blends and their nanocomposites. Therefore, 
in the present paper, an effort have been made towards the analysis of effective thermal conductivity measurement through Hot Disk Thermal Constant Analyzer based on transient plane source technique (TPS) for the thin film polymeric blends, i.e. PS/PVC, PS/PMMA and their CdS nanocomposites (PS/PVC/CdS and PS/ $\mathrm{PMMA} / \mathrm{CdS}$ ) at room temperature.

\section{Experimental}

\section{Material preparation}

To prepare polymeric blended-nanocomposite samples, firstly $\mathrm{CdS}$ nanoparticles have been prepared by simple chemical method using $\mathrm{CdCl}_{2}$ and $\mathrm{H}_{2} \mathrm{~S}$ gas produced from thiourea. The samples of PS/PMMA/CdS and PS/ $\mathrm{PVC} / \mathrm{CdS}$ polymeric nanocomposite have been prepared by dispersing prepared $\mathrm{CdS}$ nanoparticles during the preparation of respective polymeric blends of different concentrations $(0,25,50,75$, and $100 \%)$ through solution casting method. In this method the laboratory grade polymeric material which are to be blended are dissolved accordingly in the tetrahydrofuran (THF) solvent. Then 6 wt $\%$ CdS nano-filler particles of blending composition were dispersed in this solution. This solution was then stirred with the help of magnetic stirrer and then poured into flat-bottomed petri dishes to form film with a thickness of $\sim 0.05 \mathrm{~mm}$. The solvent is allowed to evaporate slowly over a period of $24 \mathrm{~h}$ in dry atmosphere. The so obtained film was then peeled off and dried in vacuum at $50{ }^{\circ} \mathrm{C}$, well below the boiling point of solvent to avoid bubbling, for $24 \mathrm{~h}$ to ensure the removal of the solvent (Mathur et al. 2010).

\section{SAXS measurement}

The small angle X-ray scattering measurements were performed through X'Pert Pro MPD system. The monochromatic X-ray beam of wavelength $1.5406 \mathrm{~A}^{\circ}$ (obtained from $\mathrm{Cu}-\mathrm{K} \alpha \mathrm{X}$-ray source) was used as incident beam for the measurements. The elliptical mirror with a $1 / 16^{\circ}$ divergence slit and a $1 / 32^{\circ}$ SAXS antiscatter slit is being adjusted. The diffracted beam side was equipped with the pixel detector used in receiving slit mode (one active channel) and with a programmable antiscatter slit set to $1 / 16^{\circ}$. All measurements were done in 2Theta scans with start angle: $-0.115^{\circ}$, end angle: $5.005^{\circ}$ and step size: $0.01^{\circ}$. For the determination of size, shape and distribution of particles, data files obtained from X'Pert Pro MPD system are being matched with the analysis templates that are provided with EasySAXS software.

\section{TEM measurement}

The TEM measurements were performed on Tecnai G2 30 U-Twin, operating at an accelerating voltage of $300 \mathrm{kV}$. The sample for TEM measurements has been prepared by dissolving respective Polymer/CdS nanocomposite film in THF solvent using ultrasonicator. At room temperature, a small drop of the above mentioned prepared respective solution was placed on the carbon-coated copper grid. Then the solvent is allowed to evaporate. Such a polymeric layer should be very thin to avoid artifacts in the TEM images.

Hot disk thermal constants analyzer measurements

Hot disk thermal constants analyzer based on transient plane source technique has been used to determine the thermal conductivity of prepared thin film samples of PS/ PVC, PS/PMMA polymeric blends and their CdS nanocomposites. The TPS technique for thermal conductivity and thermal diffusivity measurements has been discussed by (Gustafsson 1991). In this technique, TPS sensor serves as both heat source and temperature sensor. The thermal conductivity of thick film samples (of the microns order thickness), is determined in two steps. Firstly, the TPS sensor is sandwiched between the two ideal samples having smooth surfaces, and then this arrangement is placed between the two auxiliary metal (stainless steel) pieces. Data for the temperature increase over a given time are being recorded using the Hot Disk Thermal Constant Analyzer. Then in the second step, the experiment is repeated with the sensor sandwiched between the same two pieces of the metal and data for the temperature increase are again recorded under the same experimental conditions as performed in the first step. Employing the difference between these two temperature increases $(\Delta T)$, thickness of the film $(\Delta x)$ and power $(P)$ delivered to the sample, the effective thermal conductivity $(\lambda)$ can be determined through the following relation:

$\lambda=(P \Delta x) /(2 A \Delta T)$.

Here, the factor 2 is due to the symmetrical distribution of heat flux on both sides of the sample (Patidar and Saxena 2013).

\section{Results and discussion}

Structural and morphological analysis

The obtained small angle X-ray scattering data files of respective nanocomposite samples are matched with the analysis templates that are provided with EasySAXS software and through these the particle size distribution curves 


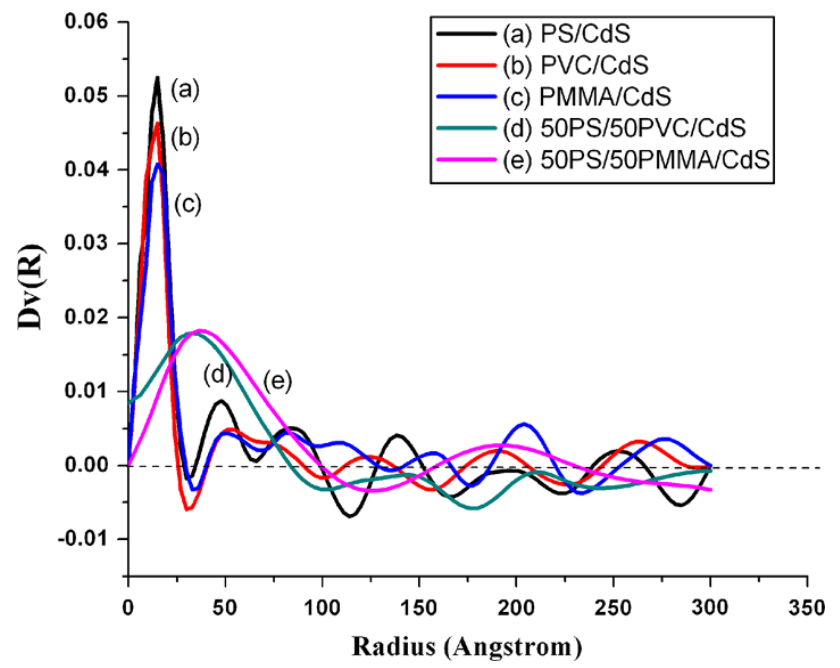

Fig. 1 Particle size distribution curves for $\mathrm{PS} / \mathrm{CdS}$, PVC/CdS, PMMA/CdS, 50PS/50PVC/CdS and 50PS/50PMMA/CdS

are plotted. Figure 1 shows the particle size distribution curves for PS/CdS, PVC/CdS, PMMA/CdS, 50PS/50PVC/ $\mathrm{CdS}$ and 50PS/50PMMA/CdS nanocomposites. The particle size distribution reports of respective nanocomposites suggest that the distribution is well approximated by a Gaussian. The minor oscillations in the distribution curve around zero towards larger particle radii may be regarded as insignificant.

In these figure curves $(\mathrm{a}-\mathrm{c})$ for the $\mathrm{PS} / \mathrm{CdS}, \mathrm{PVC} / \mathrm{CdS}$ and PMMA/CdS nanocomposites, respectively, are observed approximately centered at $R=1.3 \mathrm{~nm}$ and it means that the most frequent radius $(R)$ of $\mathrm{CdS}$ nanofillers is $1.3 \mathrm{~nm}$ and major volume fraction of these CdS nanoparticles is exhibiting radius within $0.6-2.6 \mathrm{~nm}$. Similarly from curves (d) and (e), it is revealed that $R=3.5 \mathrm{~nm}$ is as a most frequent radius of $\mathrm{CdS}$ nanofillers along with their particle size variation from $R=6.8$ to $8.8 \mathrm{~nm}$ within the 50PS/50PVC/CdS and 50PS/50PMMA/CdS nanocomposite matrices, respectively. Hence, the observed values of average particle size of CdS nanoparticles in all respective nanocomposite samples lies within nano dimensions (i.e. below $100 \mathrm{~nm}$ ) and implies that the prepared samples retain their nanocomposite nature.

Figure 2a-e shows the TEM micrographs for PS/CdS, $\mathrm{PVC} / \mathrm{CdS}$, PMMA/CdS, 50PS/50PVC/CdS and 50PS/ 50PMMA/CdS polymer nanocomposite samples, respectively. The average particle size of $\mathrm{CdS}$ nanoparticles is approximately $10 \mathrm{~nm}$. The TEM image for PS/CdS, PVC/ $\mathrm{CdS}$ and PMMA/CdS indicates that the particles are welldispersed in their respective polymer matrix with narrow size distribution whereas some clustering is observed for 50PS/50PVC/CdS and 50PS/50PMMA/CdS polymer nanocomposite sample. This is attributed due to the possibility of larger voids formation in blend matrices in comparison to pure polymer matrix. The larger voids' sites cause the higher possibility of agglomeration of $\mathrm{CdS}$ nanoparticles as observed in TEM micrographs of 50PS/ 50PVC/CdS and 50PS/50PMMA/CdS polymer nanocomposite samples.

Same fact is observed in particle size distribution curve obtained SAXS analysis for both of these samples, in terms of higher particle size of $\mathrm{CdS}$ nanoparticle. The difference between the radius of $\mathrm{CdS}$ nanoparticles obtained by SAXS and TEM is also due to the little agglomeration of $\mathrm{CdS}$ nanoparticles in respective polymer matrices at a lower weight percentage, which is not observed by SAXS measurement and is clearly indicated by TEM measurement.

\section{Thermal conductivity measurements}

Thermal conductivity of PS/PVC, PS/PMMA blends and their CdS nanocomposite thick film samples (thickness $\approx 0.1 \mathrm{~mm}$ ) have been determined through Hot disk thermal constants analyzer based on transient plane source technique with an input power of $0.5 \mathrm{~W}$ for $5 \mathrm{~s}$ at room temperature $(303 \mathrm{~K})$. Figures 3 and 4 show the thermal conductivities of PS/PVC/CdS and PS/PMMA/CdS nanocomposites as a function of weight $\%$ composition of PVC and PMMA in PS matrix, respectively.

Tables 1 and 2 depict the comparative study of thermal conductivities of prepared polymeric samples and their CdS nanocomposites. The first observation observed from the calculated results of thermal conductivity of thin film blend samples (PS/PVC and PS/PMMA) is that these values are very small in magnitude. The smaller value of thermal conductivity is due to the fact that the heat transport in solids is very sensitive to inner structure and thin films contain defects, which restrict heat transport by phonons (Guenther and Melver 1998; Cahill et al. 1989; Lambropoulos et al. 1989). This hindrance in transport of phonons leads to a lower value of thermal conductivity. Second observation is that the thermal conductivities of studied CdS nanocomposite samples are higher than their counterparts without CdS dispersed respective samples.

As we know in non-metallic material system like polymer and polymer blends, the thermal energy is mainly carried by phonons, which have a wide variation in frequency and the mean free paths. The heat carrying phonons often have large wave vectors and mean free paths in the order of nanometer range at room temperature. In this way the dimensions of the nanoparticles become comparable to the mean free paths which would lead to significant improvement in phonon transport within the nanocomposite materials. The phonon confinement and quantization 
Fig. 2 TEM micrographs of a $\mathrm{PS} / \mathrm{CdS}, \mathbf{b} \mathrm{PVC} / \mathrm{CdS}$, c PMMA/CdS, d PS/PVC/CdS and e PS/PMMA/CdS
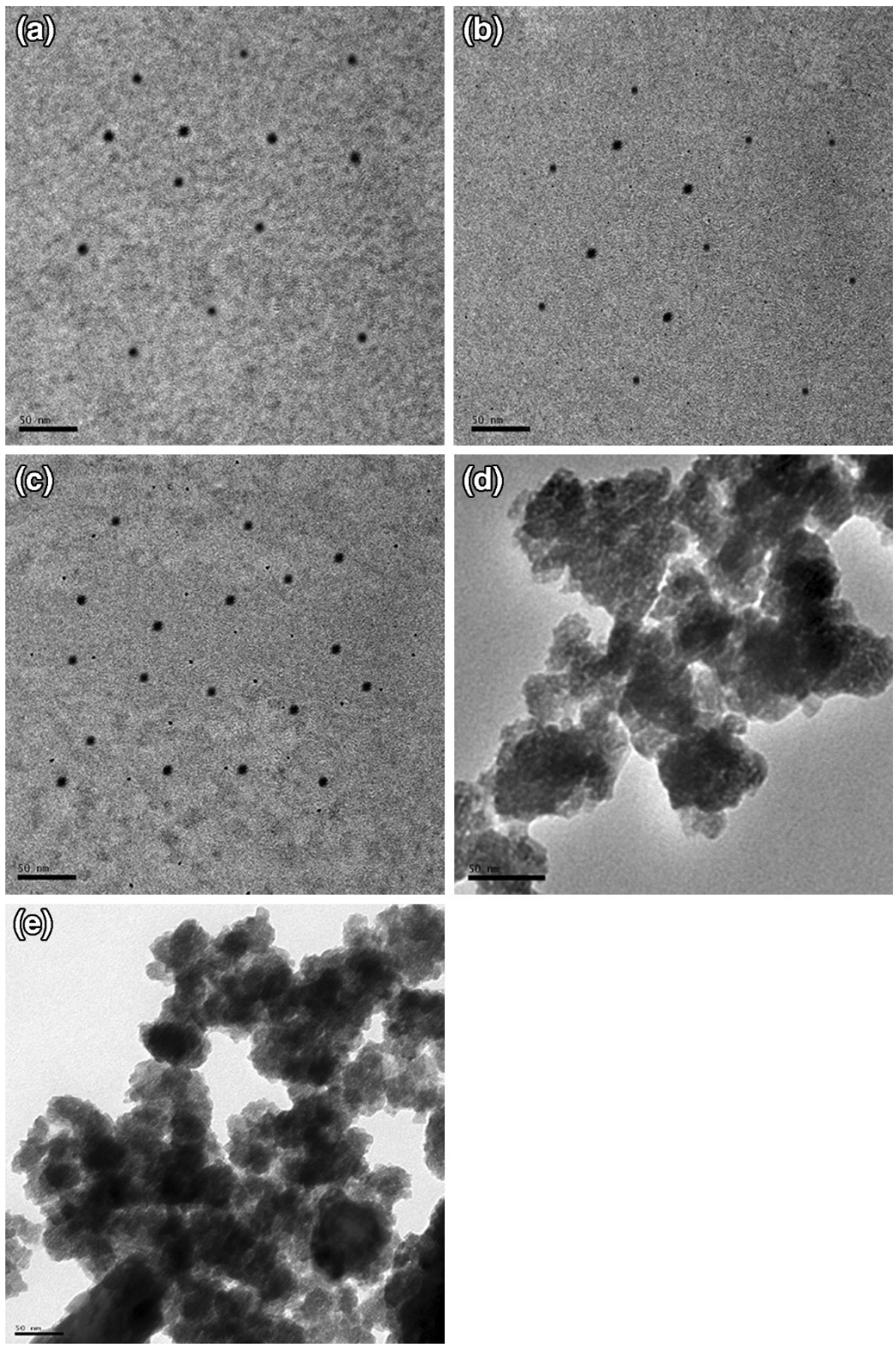

of phonon transport results modification in thermal properties. The CdS nanoparticles are semiconducting in nature with their intrinsic thermal conductivity of CdS $42.7 \mathrm{~W} /$ $\mathrm{mK}$ for $13 \mathrm{~nm}$ (Raji et al. 2004) and due to their nano dimension they can easily approach as cross-linking centers between the polymer networking. Therefore, these dispersed CdS nanoparticles promptly sub-serve for the phonon-phonon conduction in the respective polymer network. This causes the enhancement of thermal conduction in CdS nanocomposites of PS/PVC and PS/ PMMA blends.

\section{Conclusions}

The thermal conductivity of $\mathrm{CdS}$ nanocomposites of respective PS/PVC and PS/PMMA polymeric blends is found to increase due to dispersion of $\mathrm{CdS}$ nanoparticles (6 


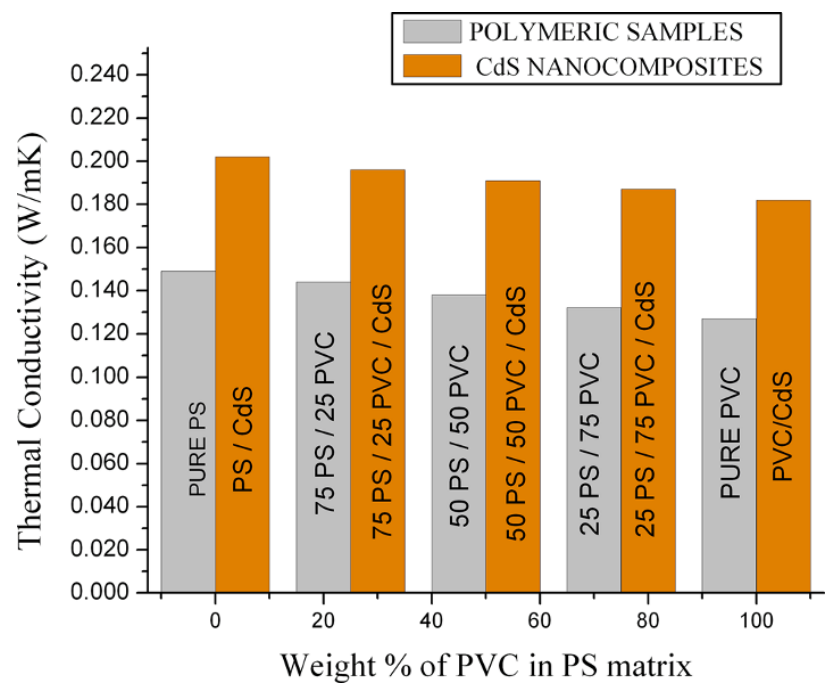

Fig. 3 Variation of thermal conductivity with weight $\%$ of PVC in $\mathrm{PS} / \mathrm{PVC} / \mathrm{CdS}$ nanocomposites

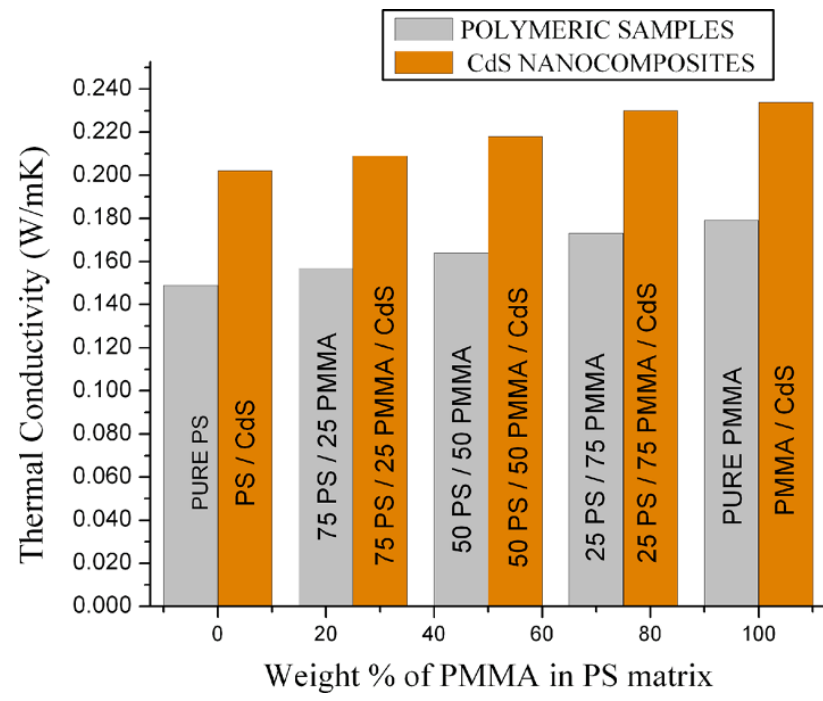

Fig. 4 Variation of thermal conductivity with weight $\%$ of PMMA in PS/PMMA/CdS nanocomposites

Table 1 Thermal conductivities of PS/PVC polymeric blends their CdS nanocomposites

\begin{tabular}{llll}
\hline $\begin{array}{l}\text { Pure and } \\
\text { blend } \\
\text { samples }\end{array}$ & $\begin{array}{l}\text { Thermal } \\
\text { conductivity } \\
(\mathrm{W} / \mathrm{mK})\end{array}$ & $\begin{array}{l}\text { Nanocomposite } \\
\text { samples }\end{array}$ & $\begin{array}{l}\text { Thermal } \\
\text { conductivity } \\
(\mathrm{W} / \mathrm{mK})\end{array}$ \\
\hline Pure PS & 0.149 & $\mathrm{PS} / \mathrm{CdS}$ & 0.202 \\
75PS/25PVC & 0.144 & 75PS/25PVC/CdS & 0.196 \\
50PS/50PVC & 0.138 & 50PS/50PVC/CdS & 0.191 \\
25PS/75PVC & 0.132 & 250PS/75PVC/CdS & 0.187 \\
Pure PVC & 0.137 & PVC/CdS & 0.191 \\
\hline
\end{tabular}

Table 2 Thermal conductivities of PS/PMMA polymeric blends their CdS nanocomposites

\begin{tabular}{llll}
\hline $\begin{array}{l}\text { Pure and } \\
\text { blend } \\
\text { samples }\end{array}$ & $\begin{array}{l}\text { Thermal } \\
\text { conductivity } \\
\text { (W/mK) }\end{array}$ & $\begin{array}{l}\text { Nanocomposite } \\
\text { samples }\end{array}$ & $\begin{array}{l}\text { Thermal } \\
\text { conductivity } \\
\text { (W/mK) }\end{array}$ \\
\hline Pure PS & 0.149 & PS/CdS & 0.202 \\
75PS/25PMMA & 0.159 & 75PS/25PMMA/CdS & 0.211 \\
50PS/50PMMA & 0.164 & 50PS/50PMMA/CdS & 0.218 \\
25PS/75PMMA & 0.171 & 25PS/75PMMA/CdS & 0.228 \\
Pure PMMA & 0.179 & PMMA/CdS & 0.234 \\
\hline
\end{tabular}

wt $\%)$. These dispersed CdS nanoparticles having higher thermal conductivity and form a bridged polymer network leading to the increase in the phonon-phonon interaction within the respective nanocomposite matrix.

Open Access This article is distributed under the terms of the Creative Commons Attribution License which permits any use, distribution, and reproduction in any medium, provided the original author(s) and the source are credited.

\section{References}

Agari Y, Shimada M, Veda A (1997a) Thermal-diffusivity and conductivity of PS PPO blends. Polymer 38:2649-2655

Agari Y, Ueda A, Omura Y, Nagai S (1997b) Thermal-diffusivity and conductivity of PMMA PC blends. Polymer 38:801-807

Cahill DG, Fischer E, Klitsner T, Swartz ET, Pohl RO (1989) Thermal conductivity of thin films: measurements and understanding. J Vac Sci Technol A 7:1259-1266

Chen L, Zhu J, Qing L, Chen S, Wang Y (2007) Controllable synthesis of functionalized CdS nanocrystals and CdS/PMMA nanocomposite hybrids. Eur Polymer J 43:4593-4601

Guenther AH, Melver JK (1998) The role of thermal conductivity in the pulsed laser damage sensitivity of optical thin films. Thin Solids Films 163:203-214

Gustafsson GE (1991) Transient plane source techniques for thermal conductivity and thermal diffusivity measurements of solid materials. Rev Scientific Instrum 62:797-804

Jung-Chul L, Wonjoo L, Sung-Hwan H, Tae Geun K, Yun-Mo S (2009) Synthesis of hybrid solar cells using CdS nanowire array grown on conductive glass substrates. Electrochem Commun 11:231-234

Lambropoulos JC, Jolly MR, Amsden CA, Gilman SE, Sinicropi MJ, Diakomihalis D, Jacobs SD (1989) Thermal conductivity of dielectric thin films. J Appl Phys 66:4230-4242

Mathur V, Sharma K (2013) Evaluation of morphological effect on thermal and mechanical performance of PS/PMMA/CdS nanocomposite systems. Adv Nanopart 2:205-216

Mathur V, Dixit M, Rathore KS, Saxena NS, Sharma K (2009) Morphological effects on mechanical properties of polystyrenepolyvinylchloride blends. Ph Transit 82:769-779

Mathur V, Dixit M, Saxena NS, Sharma K (2010) Phase transition and mechanical properties of $\mathrm{ps} / \mathrm{pvc} / \mathrm{cds}$ polymeric nanocomposites. AIP Proc 1249:141-144

Mathur V, Rathore KS, Sharma K (2013) Evaluation of energy band gap, thermal conductivity, phase transition temperature and 
elastic response of ps/cds semiconducting optical nanocomposite. World J Nano Sci Eng 3:93-99

Patidar D, Saxena NS (2013) Influence of CdS nano additives on the thermal conductivity of Poly(vinyl chloride)/CdS nanocomposites. Adv Nanopart 2:11-15
Raji P, Sanjeeviraja C, Ramachandram K (2004) Thermal properties of nano-crystalline CdS. Cryst Res Technol 39:617-622

Rong MZ, Zhang MQ, Liang HC, Zeng HM (2003) Surface modification and particles size distribution control in nanoCdS/polystyrene composite film. Chem Phys 286:267-276 